Open Access

\title{
Correction to: Bitcoin and the rise of decentralized autonomous organizations
}

Ying-Ying Hsieh ${ }^{1 *}$, Jean-Philippe Vergne ${ }^{2}$, Philip Anderson ${ }^{3}$, Karim Lakhani ${ }^{4}$ and Markus Reitzig ${ }^{5}$

* Correspondence:

y.hsieh@imperial.ac.uk

${ }^{1}$ Imperial College Business School,

Imperial College London, South Kensington Campus, Exhibition

Road, London SW7 2AZ, UK

Full list of author information is

available at the end of the article

\section{Correction to: J Organ Des}

https://doi.org/10.1186/s41469-018-0038-1

Following the publication of this article (Hsieh 2018), it was noted that the author list was incomplete and was missing the following three authors:

- Philip Anderson

- Karim Lakhani

- Markus Reitzig

This was due to a typesetting error and the publisher apologizes to the authors and readers for any inconvenience caused. The original article has been updated.

\section{Author details}

'Imperial College Business School, Imperial College London, South Kensington Campus, Exhibition Road, London SW7 2AZ, UK. ${ }^{2}$ Ivey Business School, Western University, 1255 Western Road, London, ON N6G ON1, Canada. ${ }^{3}$ INSEAD, 1 Ayer Rajah Avenue, Singapore 138676, Singapore. ${ }^{4}$ Harvard Business School, Morgan Hall 419 Harvard Business School Soldiers Field Rd., Boston, MA 02163, USA. ${ }^{5}$ Department of Business Administration Strategic Management Subject Area, University of Vienna, Oskar-Morgenstern-Platz 1, Vienna 1090, Austria.

Received: 4 January 2019 Accepted: 8 January 2019

Published online: 12 February 2019

\section{Reference}

Hsieh et al (2018) Bitcoin and the rise of decentralized autonomous organizations. J Organ Des 7:14. https://doi.org/ 10.1186/s41469-018-0038-1 